\title{
FUNCTIONALISED ORGANOSOLV LIGNINS SUITABLE FOR MODIFICATIONS OF HARD SURFACES
}

\section{SUPPORTING INFORMATION}

Paola Giannì ${ }^{\mathrm{a}}$, Heiko Lange $\mathrm{b}^{\mathrm{b}, \mathrm{c}, \dagger, *}$, Claudia Crestini ${ }^{\mathrm{c}, \mathrm{d}, \grave{\dagger}, *}$

a: Department of Chemical Sciences and Technologies, University of Rome 'Tor Vergata', Via della Ricerca Scientifica, 00133 Rome, Italy

b: Department of Pharmacy, University of Naples 'Federico II', Via Domenico Montesano 49, 80131 Naples, Italy

c: CSGI - Center for Colloid and Surface Science, Via della Lastruccia 3, 50019 Sesto Fiorentino, Italy

d; Department of Molecular Science and Nanosystems, University of Venice Ca' Foscari, Via Torino 155, 30170 Venice Mestre, Italy

$\dagger$ : Also affiliated with a) via NAST - Nanoscience \& Nanotechnology \& Innovative Instrumentation Center.

* Corresponding authors: heiko.lange@unina.it claudia.crestini@unive.it 
Table S1: Representative ${ }^{31} \mathrm{P}$ NMR spectra of realised functionalised WS-OSL and WS-OSLbased copolymer functionalised either chemically or via enzymatic catalysis as described in the manuscript.

WS-OSL (starting material)

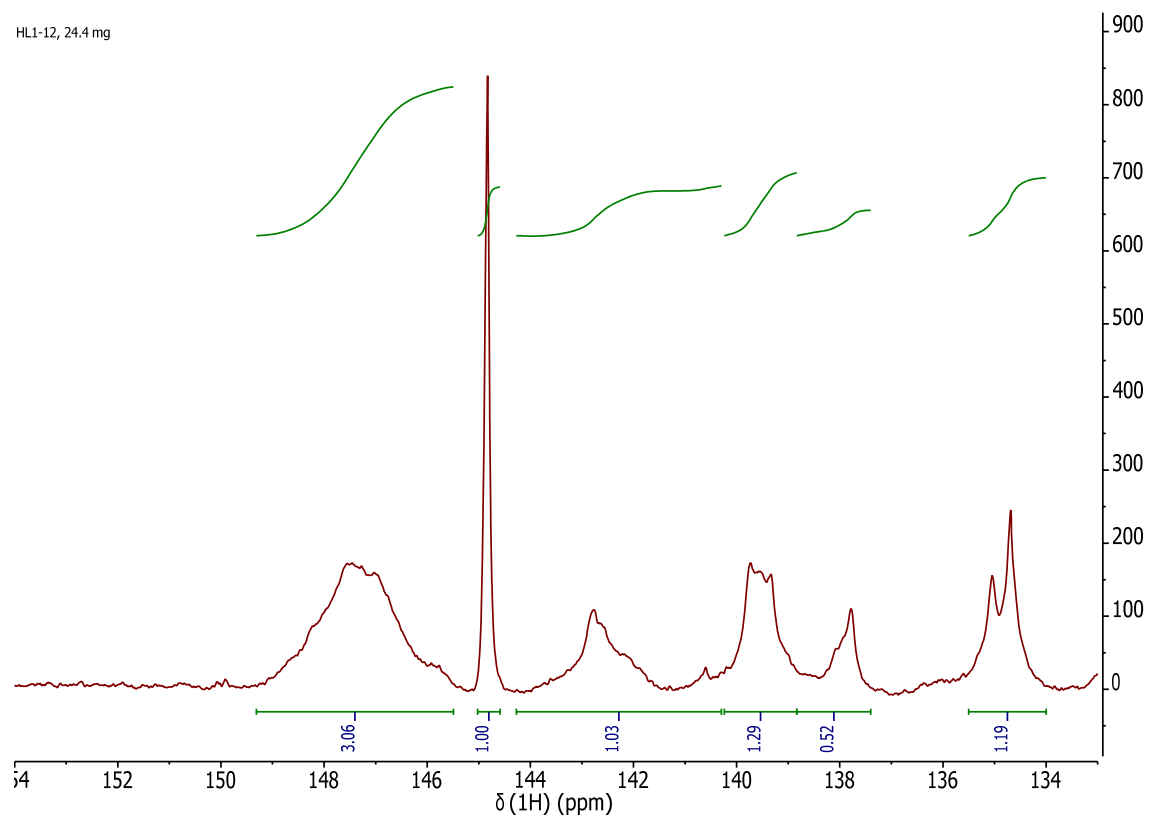

WS-OSL blank

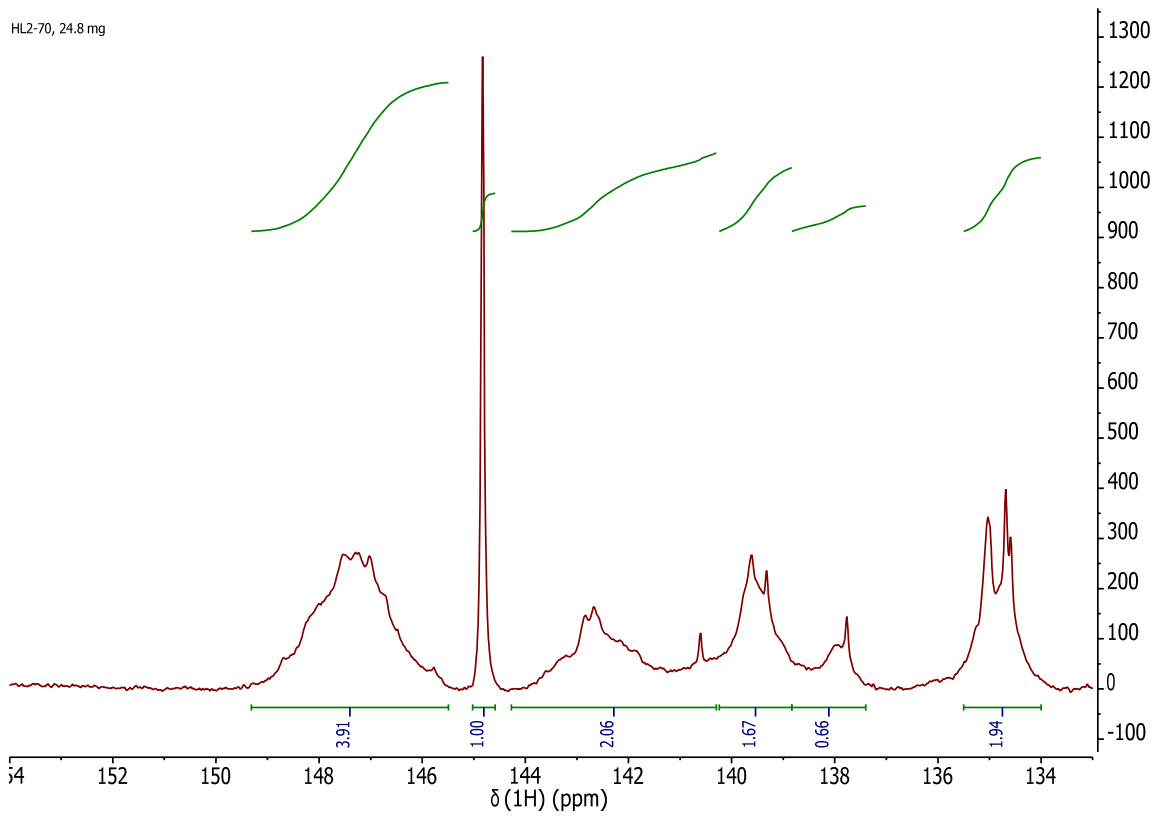


Table S1 (continued): Representative ${ }^{31} \mathrm{P}$ NMR spectra for realised functionalised WS-OSL and WS-OSL-based copolymer functionalised either chemically or via enzymatic catalysis as described in the manuscript.

WS-OSL + $\mathrm{C}_{3}-\mathrm{NMe}_{3} \mathrm{Cl}$ (2.0 eq.) [Table 1, entry 4]

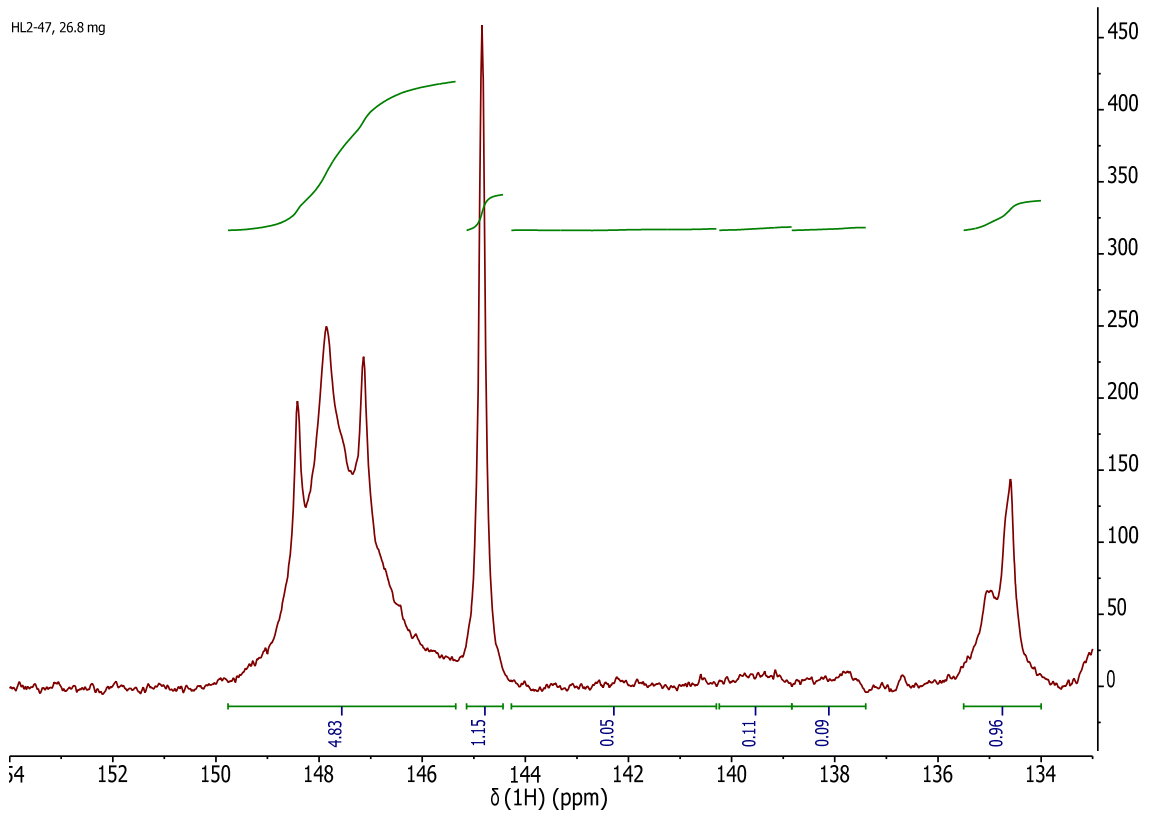

WS-OSL + $\mathrm{C}_{3}-\mathrm{NMe}_{3} \mathrm{Cl}$ (1.0 eq.) [Table 1, entry 5]

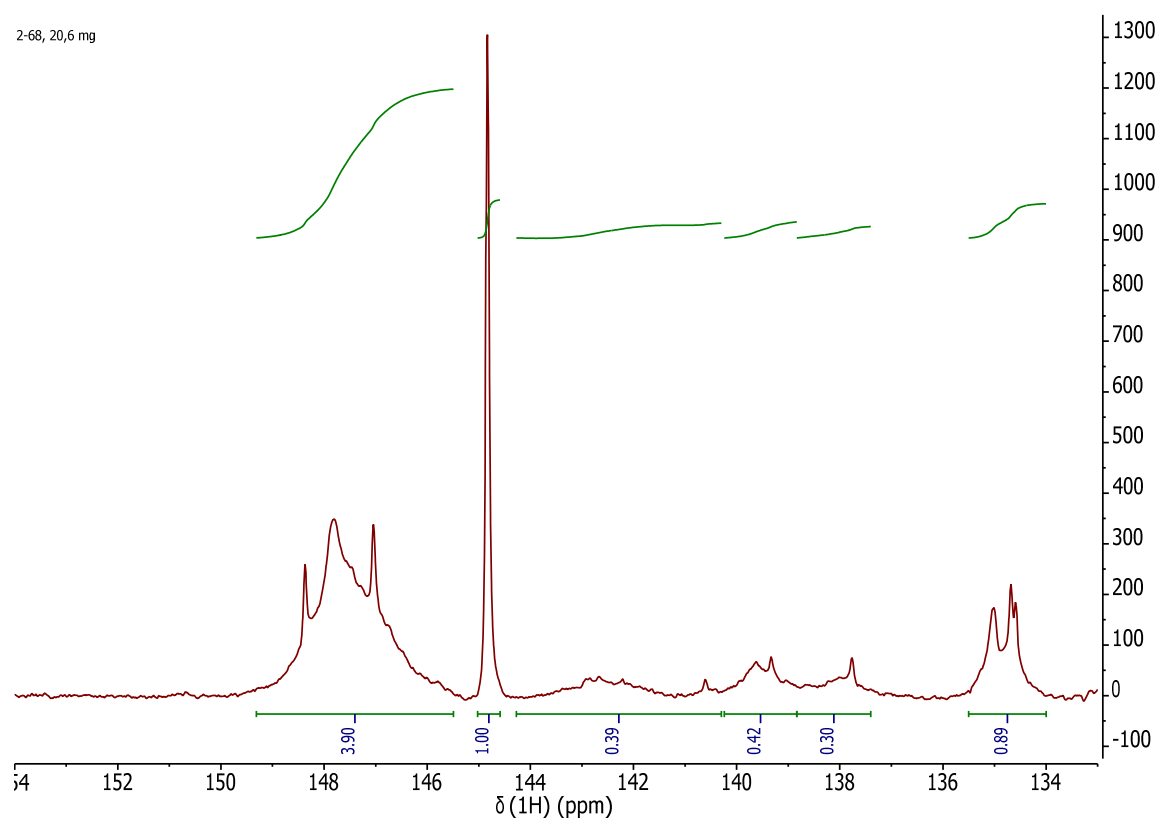


Table S1 (continued): Representative ${ }^{31} \mathrm{P}$ NMR spectra for realised functionalised WS-OSL and WS-OSL-based copolymer functionalised either chemically or via enzymatic catalysis as described in the manuscript.

WS-OSL + $\mathrm{C}_{9}-\mathrm{NMe}_{3} \mathrm{Cl}$ (2.0 eq.) [Table 1, entry 6]

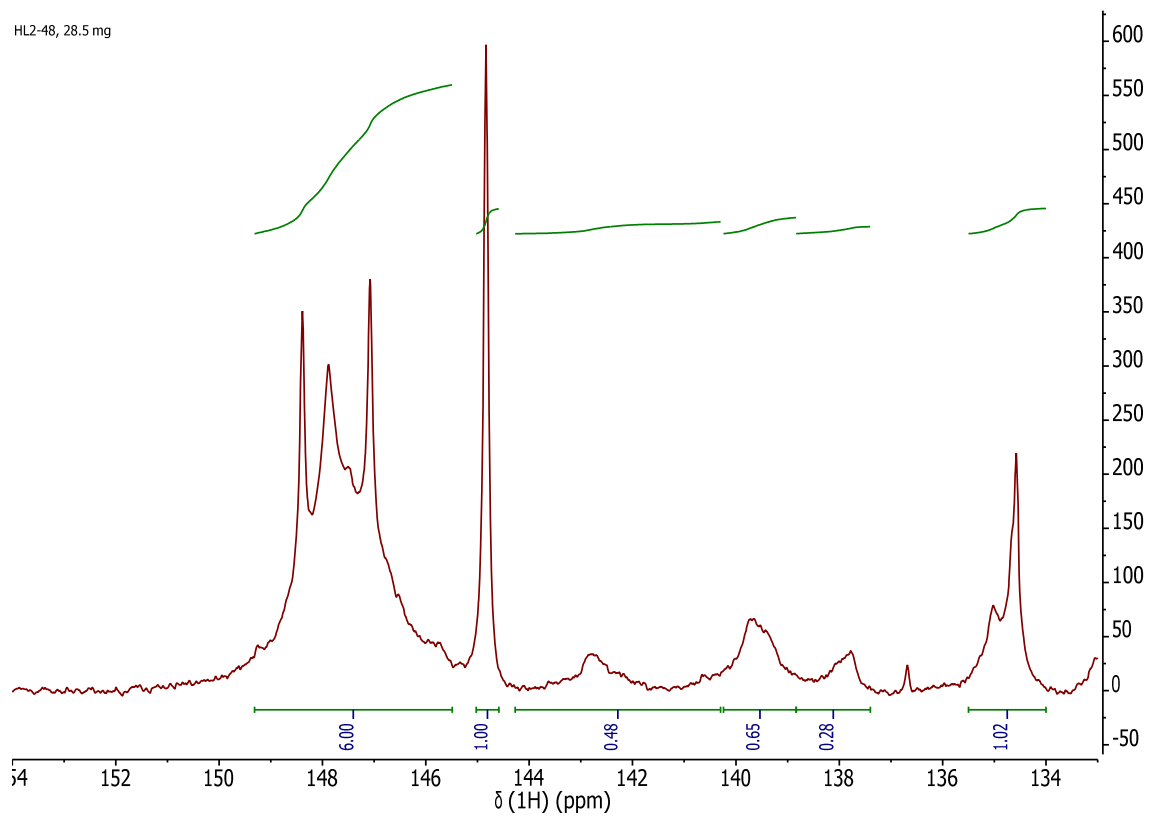

WS-OSL $+\mathrm{C}_{3}-\mathrm{CO}_{2} \mathrm{H}(1.0$ eq.) [Table 1, entry 7]

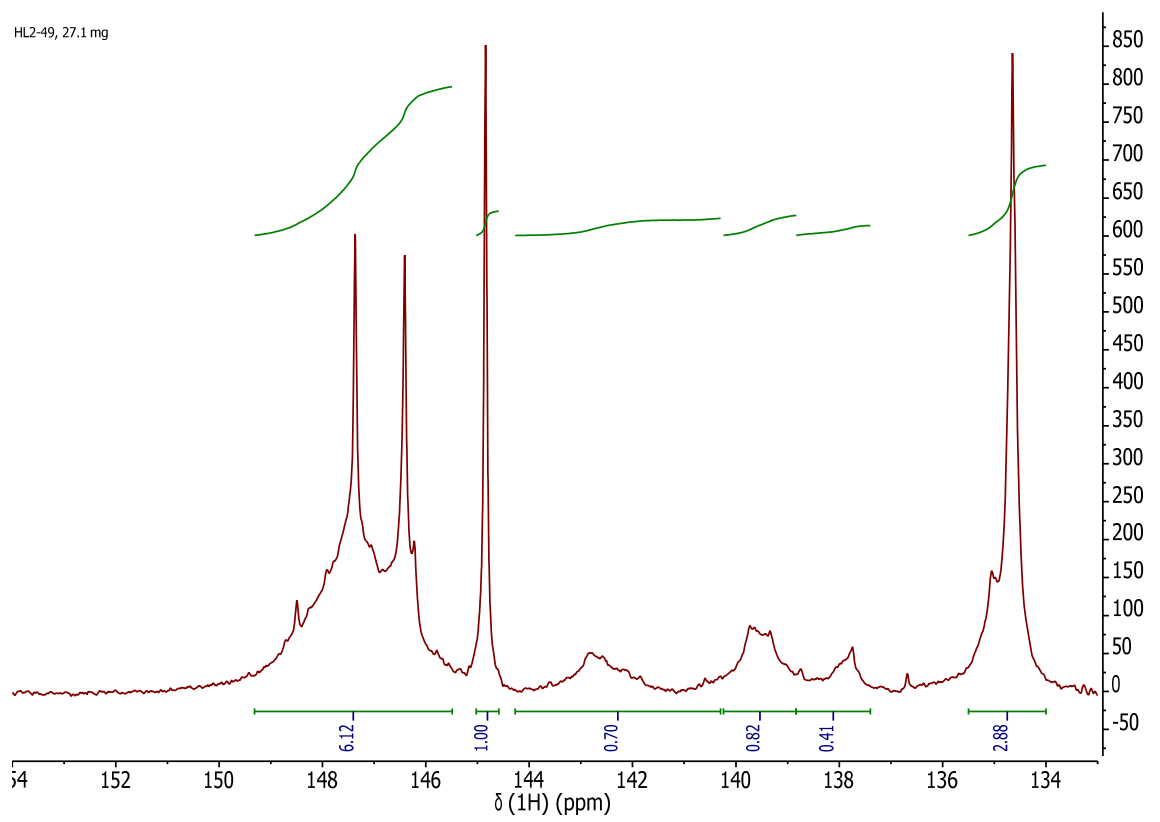


Table S1 (continued): Representative ${ }^{31} \mathrm{P}$ NMR spectra for realised functionalised WS-OSL and WS-OSL-based copolymer functionalised either chemically or via enzymatic catalysis as described in the manuscript.

WS-OSL $+\mathrm{C}_{2}-\mathrm{CO}_{2} \mathrm{H}(2.0$ eq.) [Table 1, entry 9]

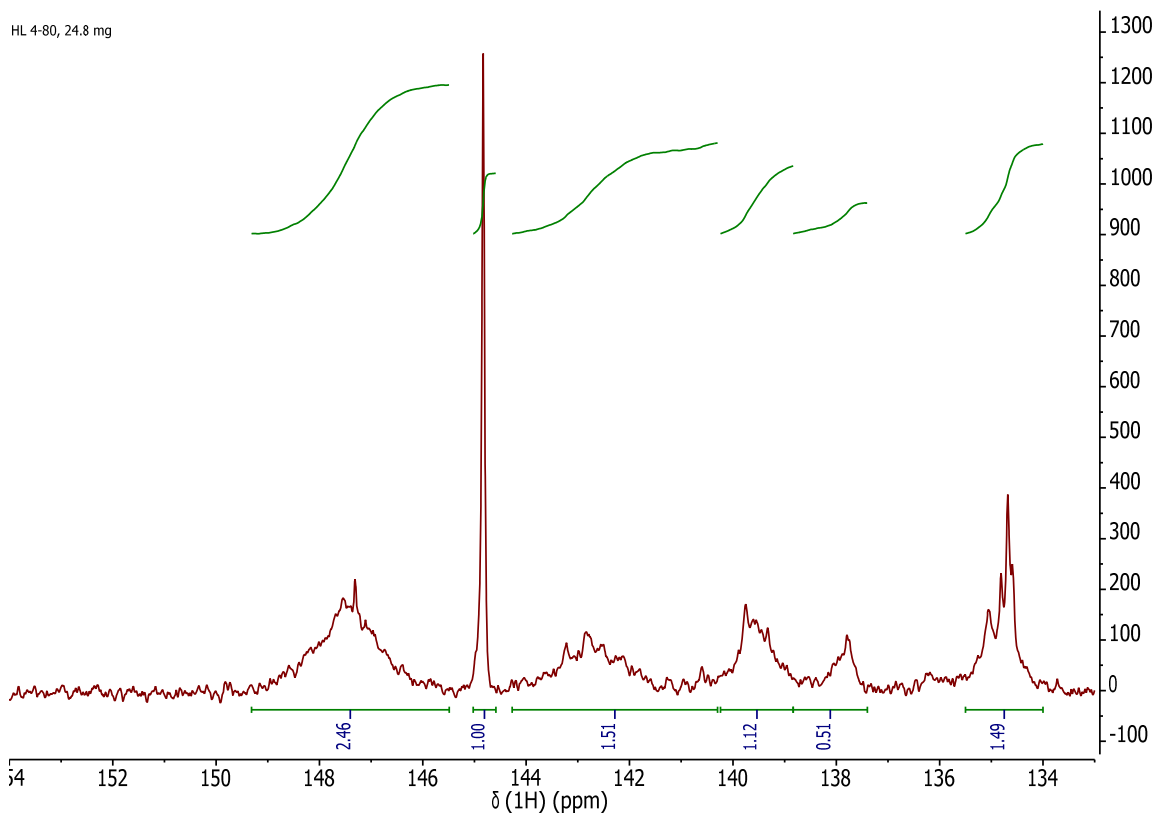

WS-OSL $+\mathrm{C}_{8}-\mathrm{CO}_{2} \mathrm{H}(2.0$ eq.) [Table 1, entry 10]

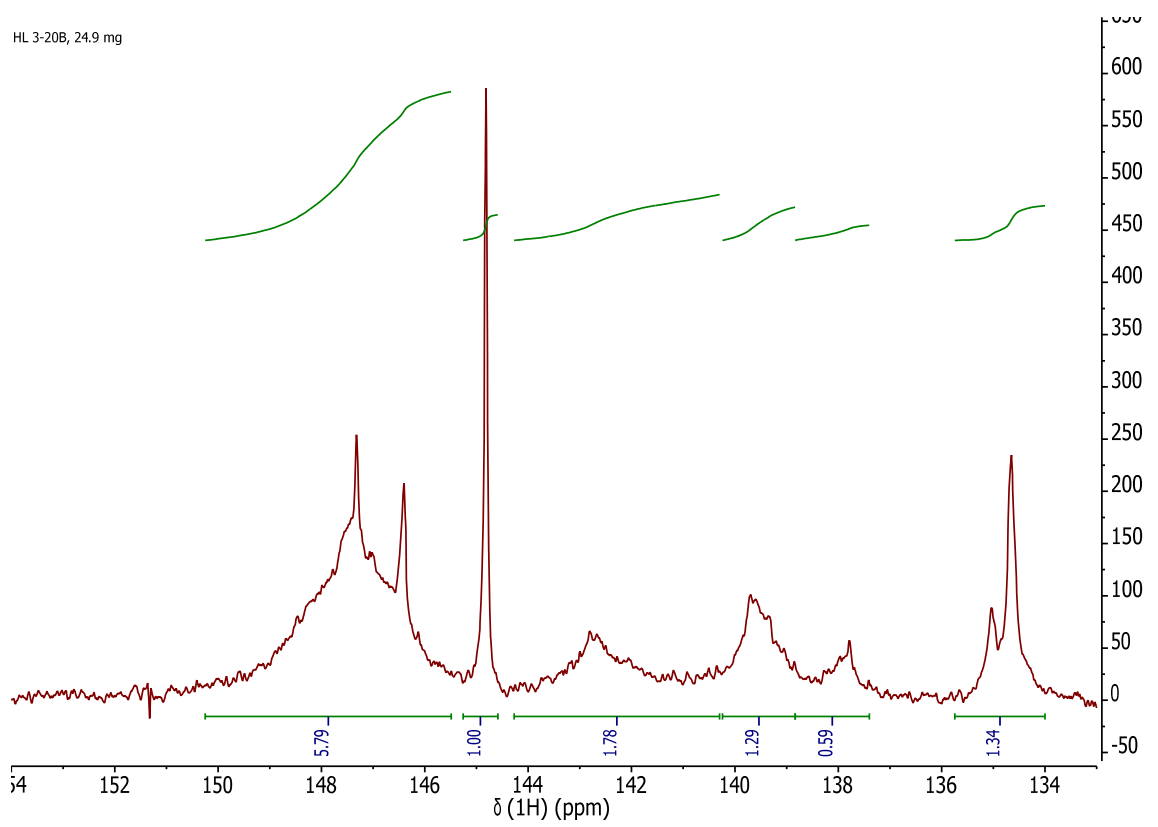


Table S1 (continued): Representative ${ }^{31} \mathrm{P}$ NMR spectra for realised functionalised WS-OSL and WS-OSL-based copolymer functionalised either chemically or via enzymatic catalysis as described in the manuscript.

WS-OSL $+\mathrm{C}_{3}-\mathrm{CO}_{2} \mathrm{H}(0.5$ eq. $)+\mathrm{C}_{3}-\mathrm{NMe}_{3} \mathrm{Cl}(0.5$ eq.) [Table 1, entry 11]

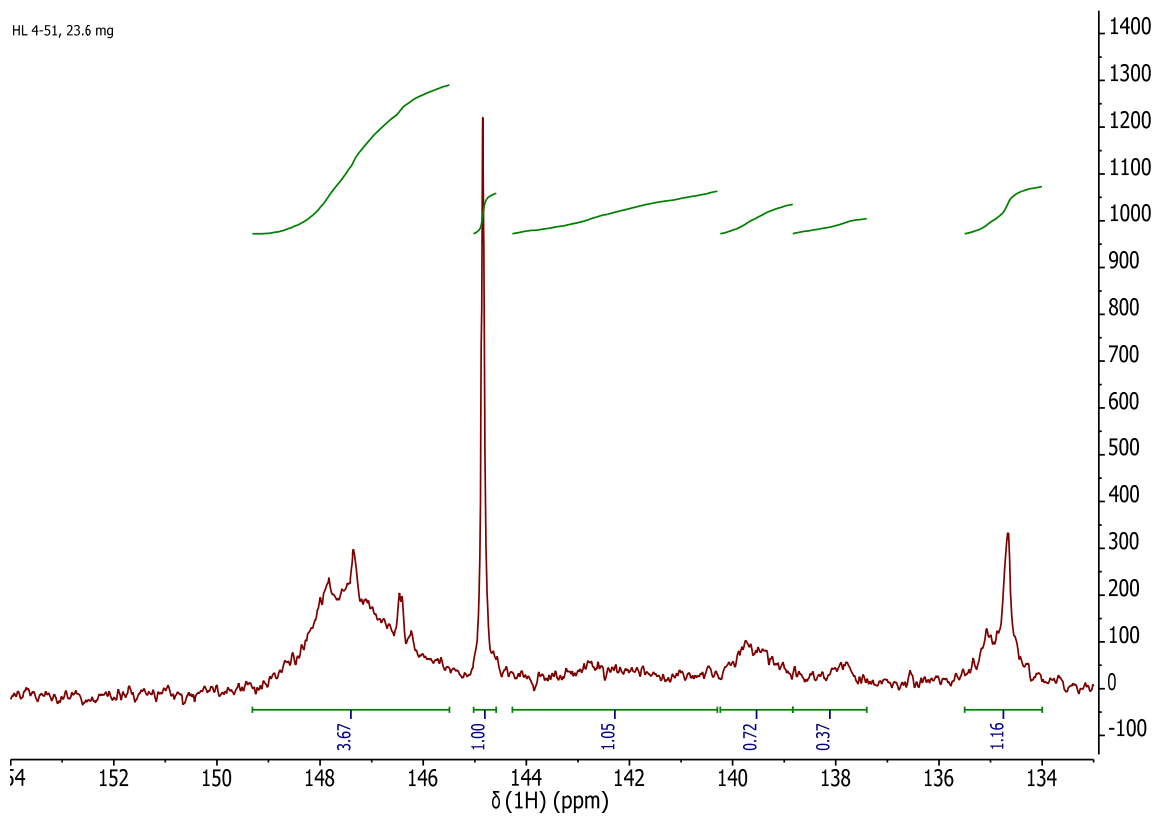

WS-OSL + PDMS 800 (5.0 eq.) [Table 2, entry 4]

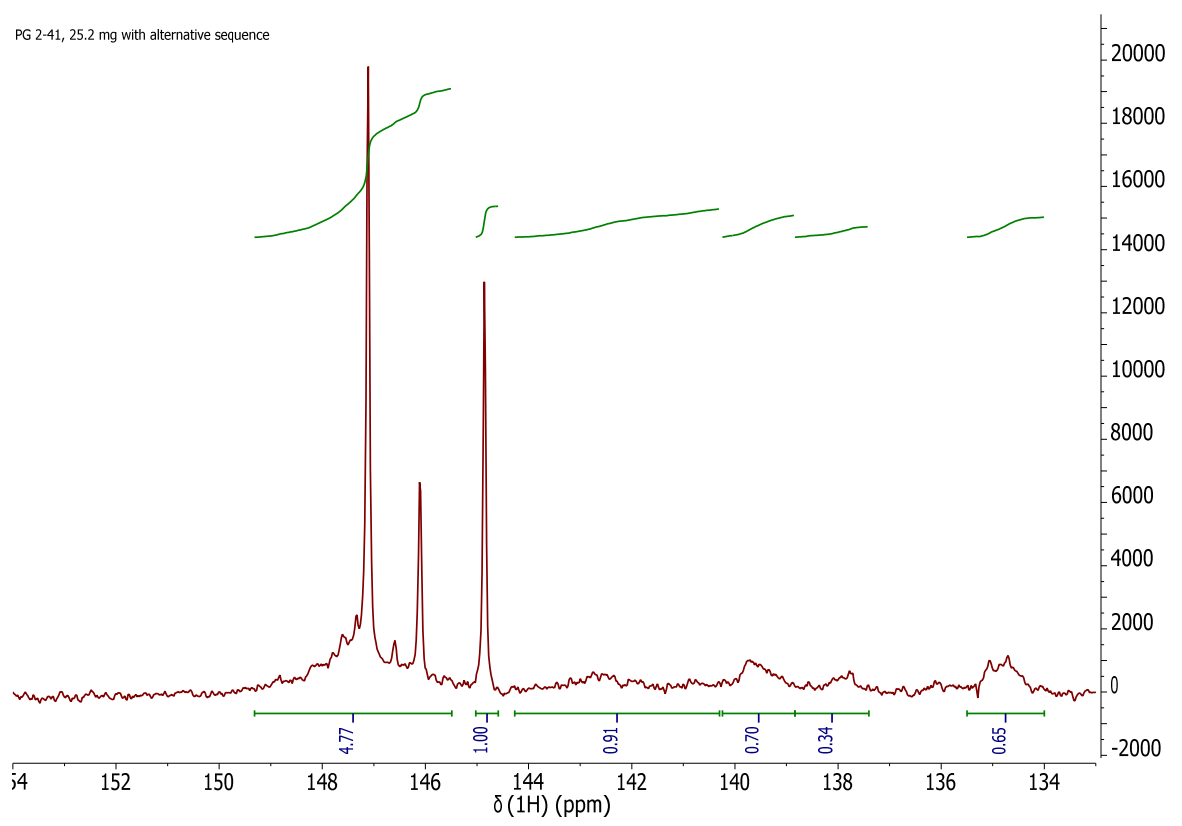


Table S1 (continued): Representative ${ }^{31} \mathrm{P}$ NMR spectra for realised functionalised WS-OSL and WS-OSL-based copolymer functionalised either chemically or via enzymatic catalysis as described in the manuscript.

WS-OSL + PEG 500 (10 eq.) [Table 2, entry 7]

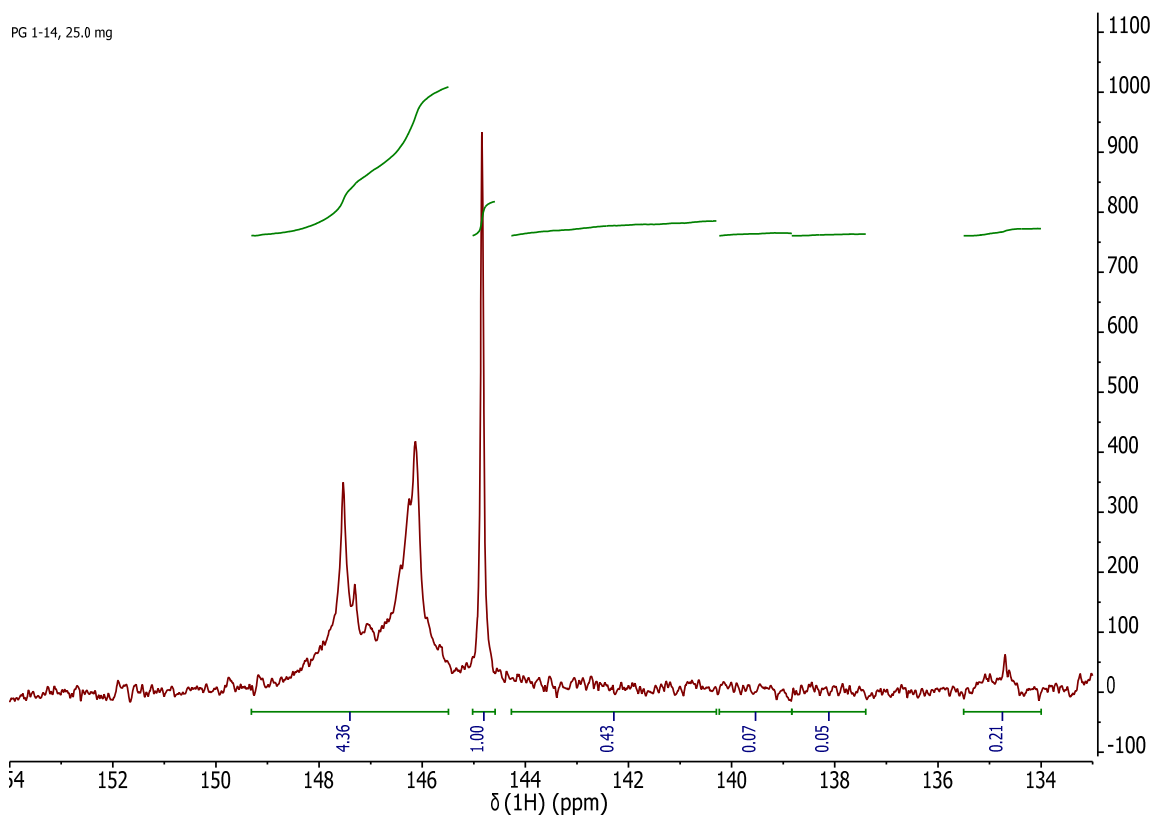

WS-OSL $+\mathrm{C}_{3}-\mathrm{NMe}_{3} \mathrm{Cl}(0.6$ eq. $)+$ PEG $_{500}(0.5$ eq.) $[$ Table 2, entry 8]

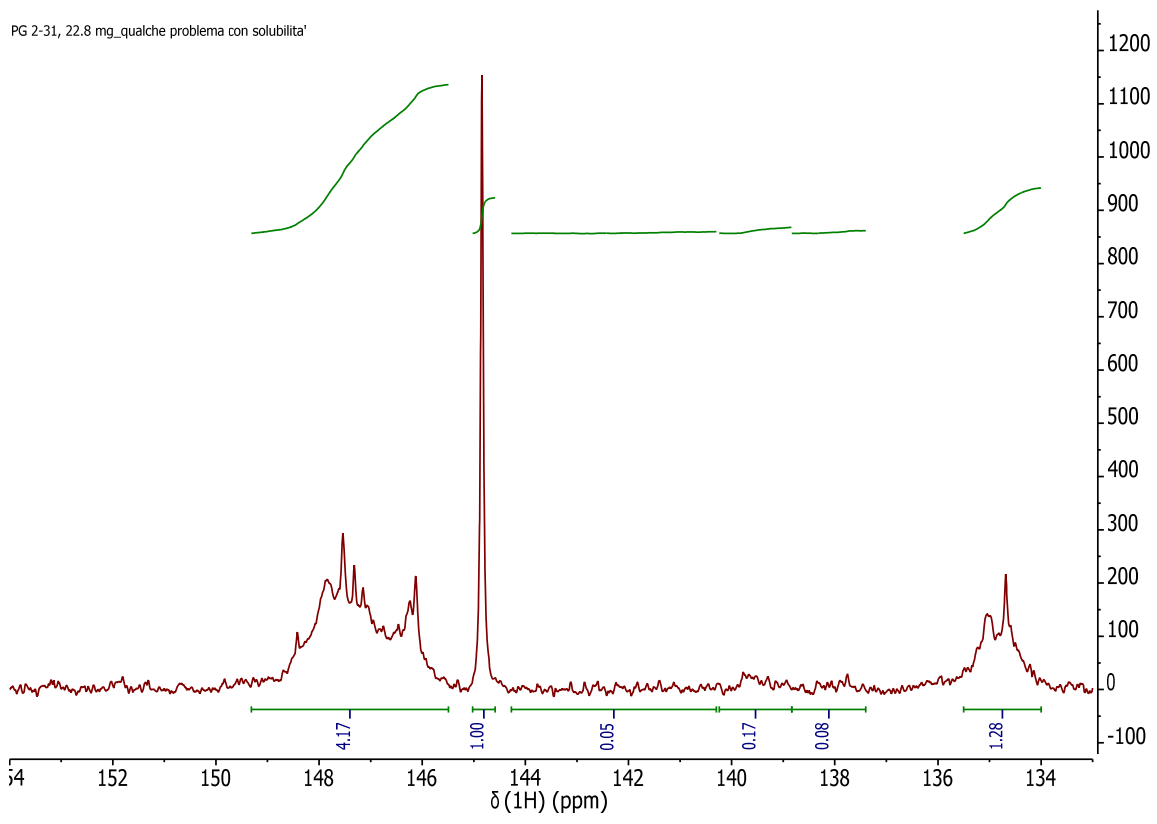


Table S1 (continued): Representative ${ }^{31} \mathrm{P}$ NMR spectra for realised functionalised WS-OSL and WS-OSL-based copolymer functionalised either chemically or via enzymatic catalysis as described in the manuscript.

WS-OSL $+\mathrm{C}_{3}-\mathrm{CO}_{2} \mathrm{H}\left(0.6\right.$ eq.) $+\mathrm{PEG}_{500}(0.5$ eq.) [Table 2, entry 9]

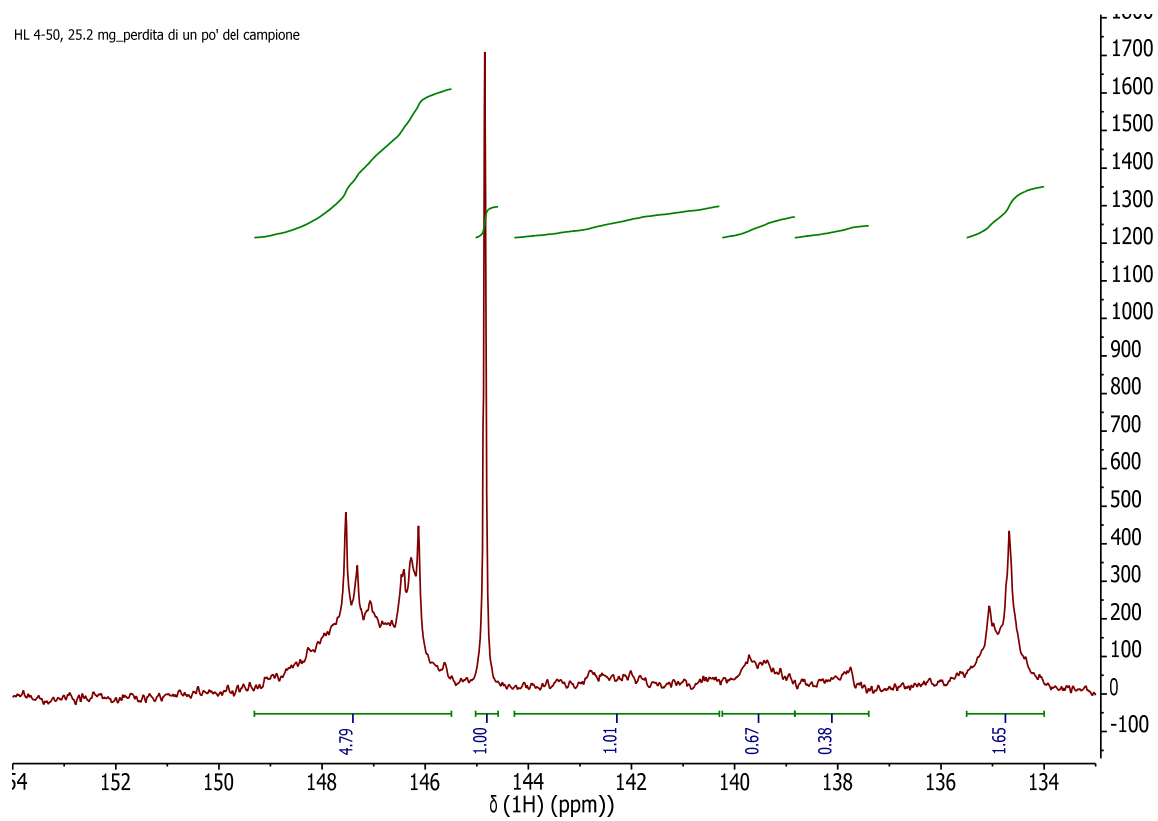

WS-OSL + PDMS 5000 (1.0 eq.) [Table 3, entry 3]

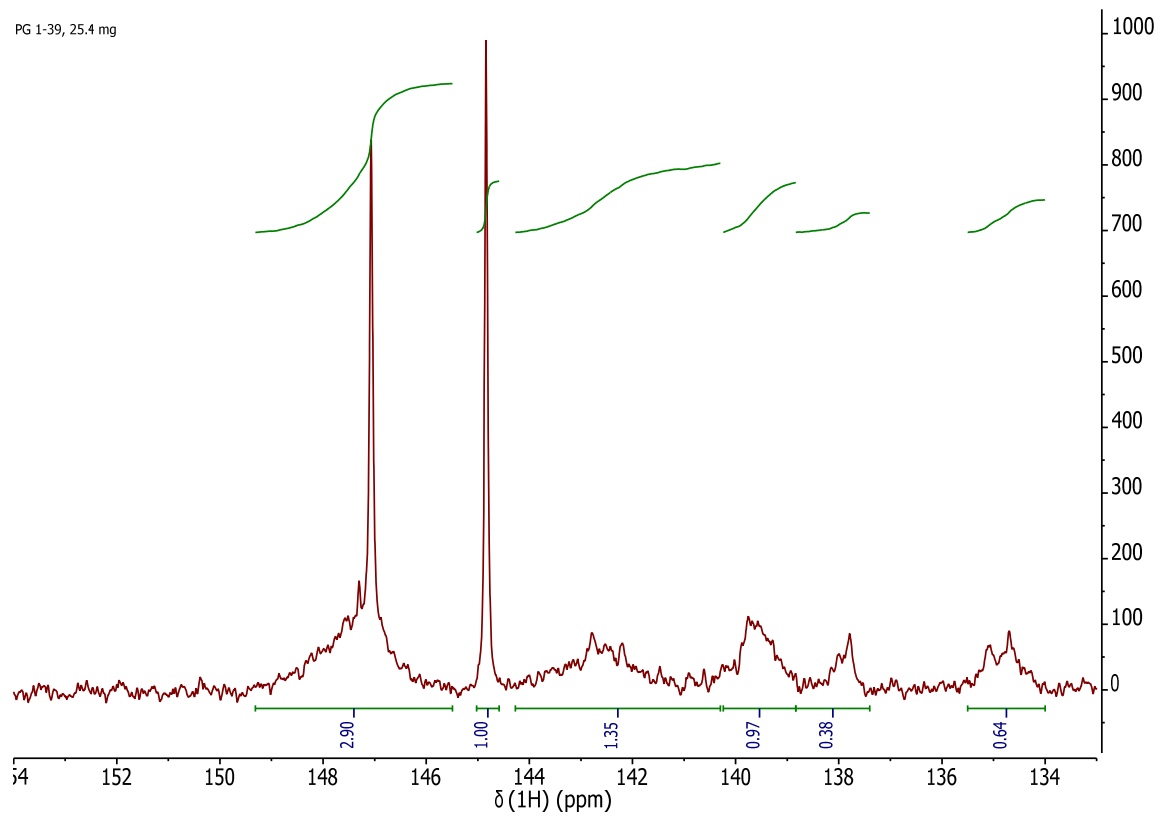


Table S1 (continued): Representative ${ }^{31} \mathrm{P}$ NMR spectra for realised functionalised WS-OSL and WS-OSL-based copolymer functionalised either chemically or via enzymatic catalysis as described in the manuscript.

WS-OSL + PDMS 800 (10 eq.) [Table 3, entry 4]

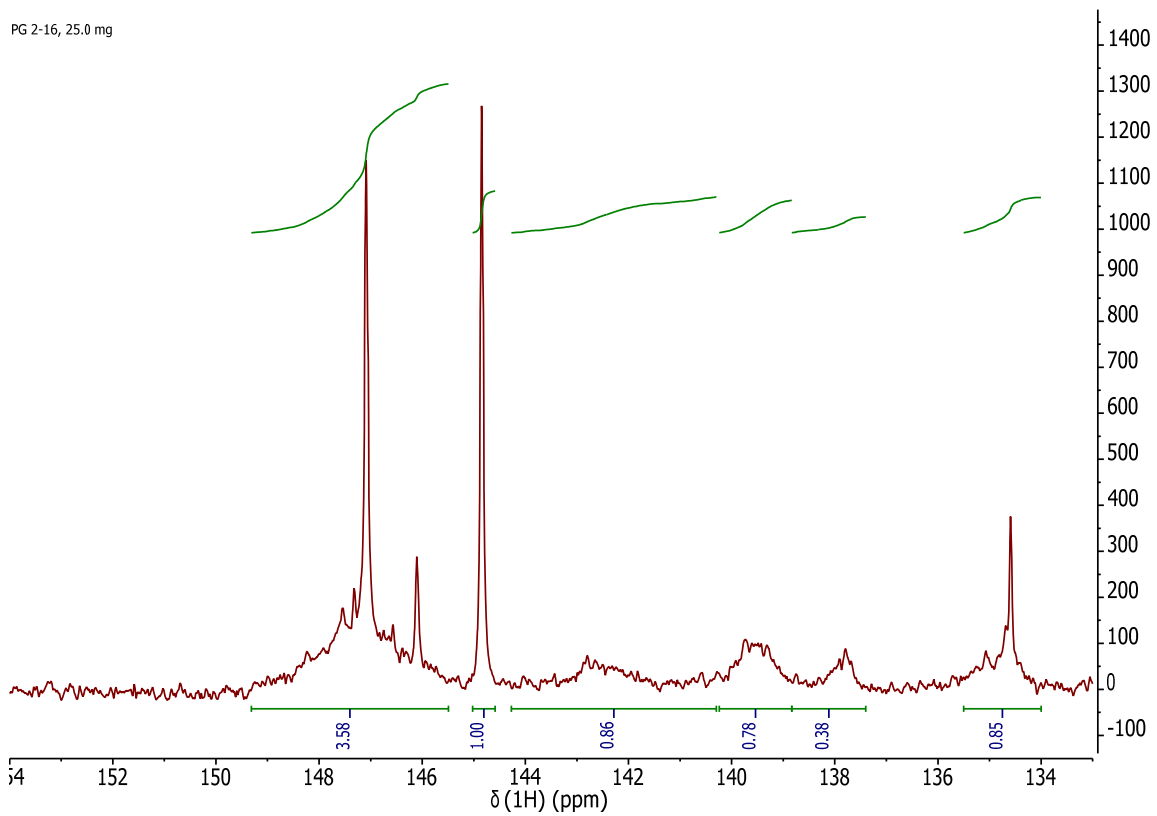

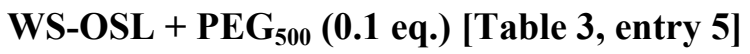

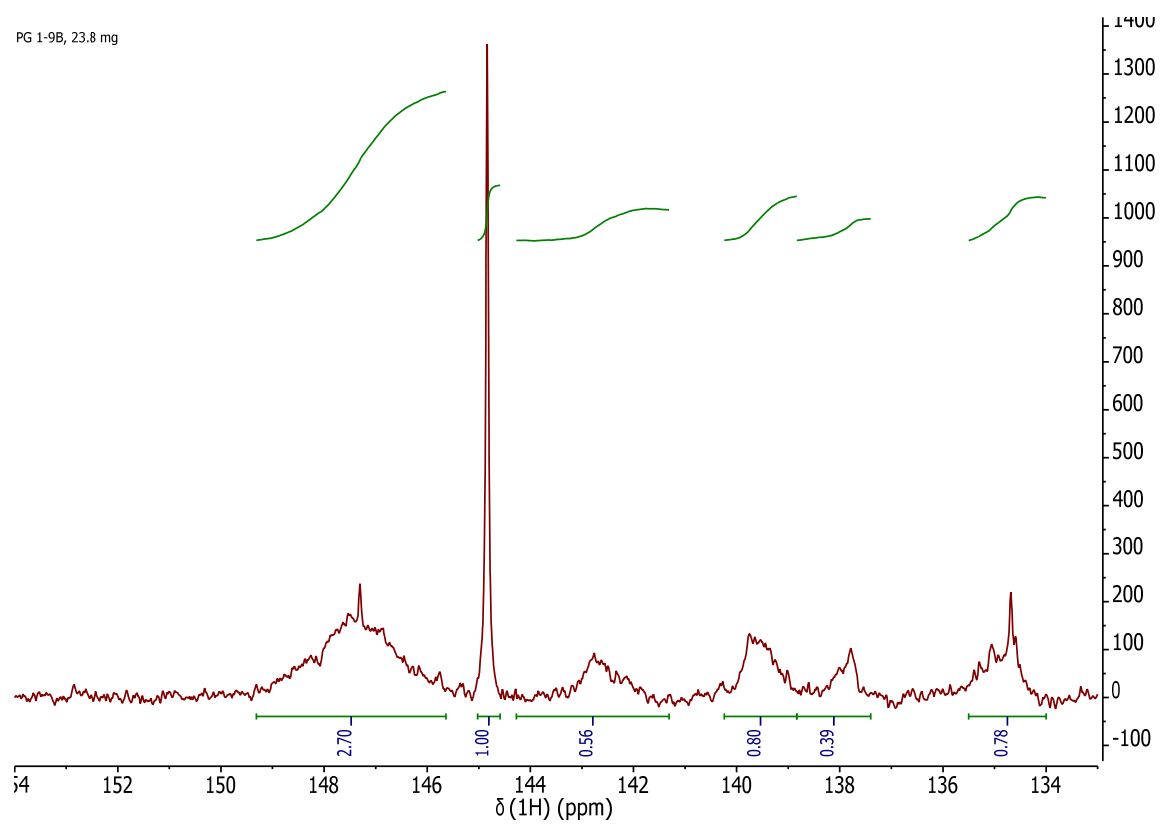


Table S1 (continued): Representative ${ }^{31} \mathrm{P}$ NMR spectra for realised functionalised WS-OSL and WS-OSL-based copolymer functionalised either chemically or via enzymatic catalysis as described in the manuscript.

WS-OSL + PEG ${ }_{500}(10$ eq.) [Table 3, entry 6]

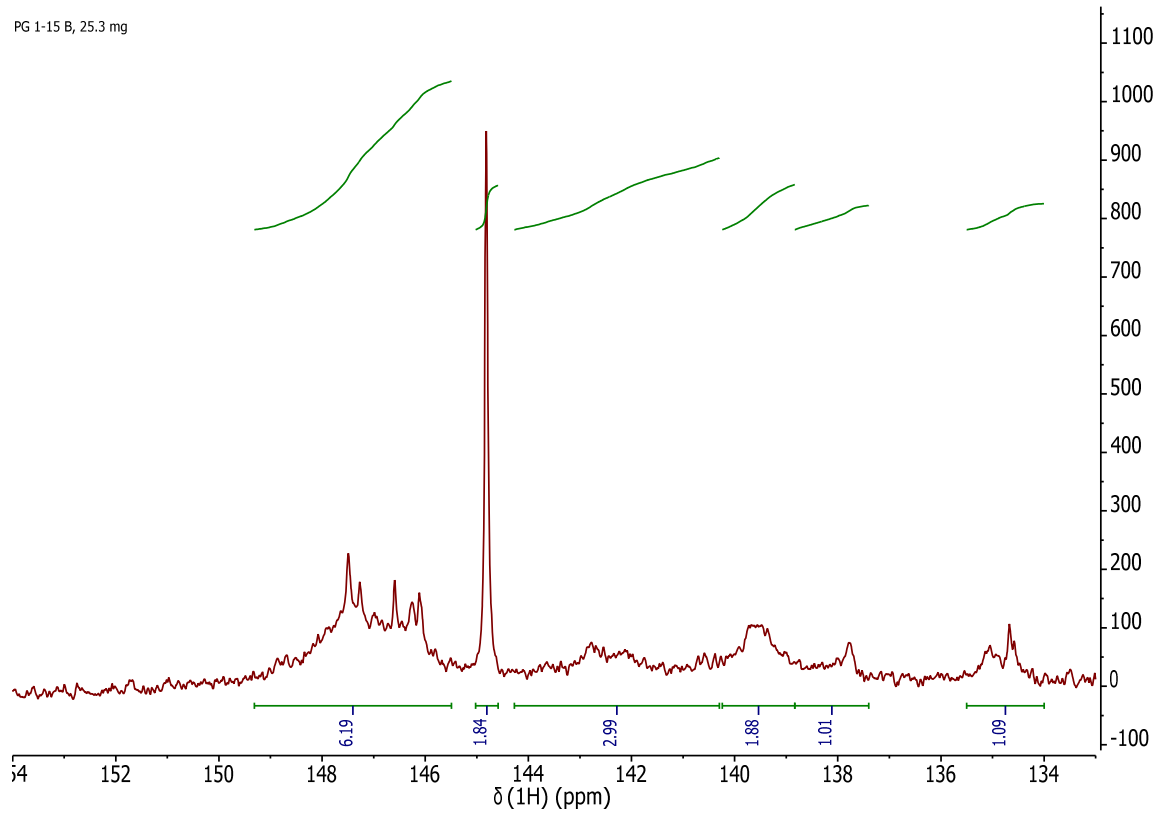


Table S2: Representative ${ }^{31}$ P NMR spectra for realised functionalised CS-OSL and CS-OSLbased copolymer functionalised either chemically or via enzymatic catalysis as described in the manuscript.

CS-OSL (starting material)

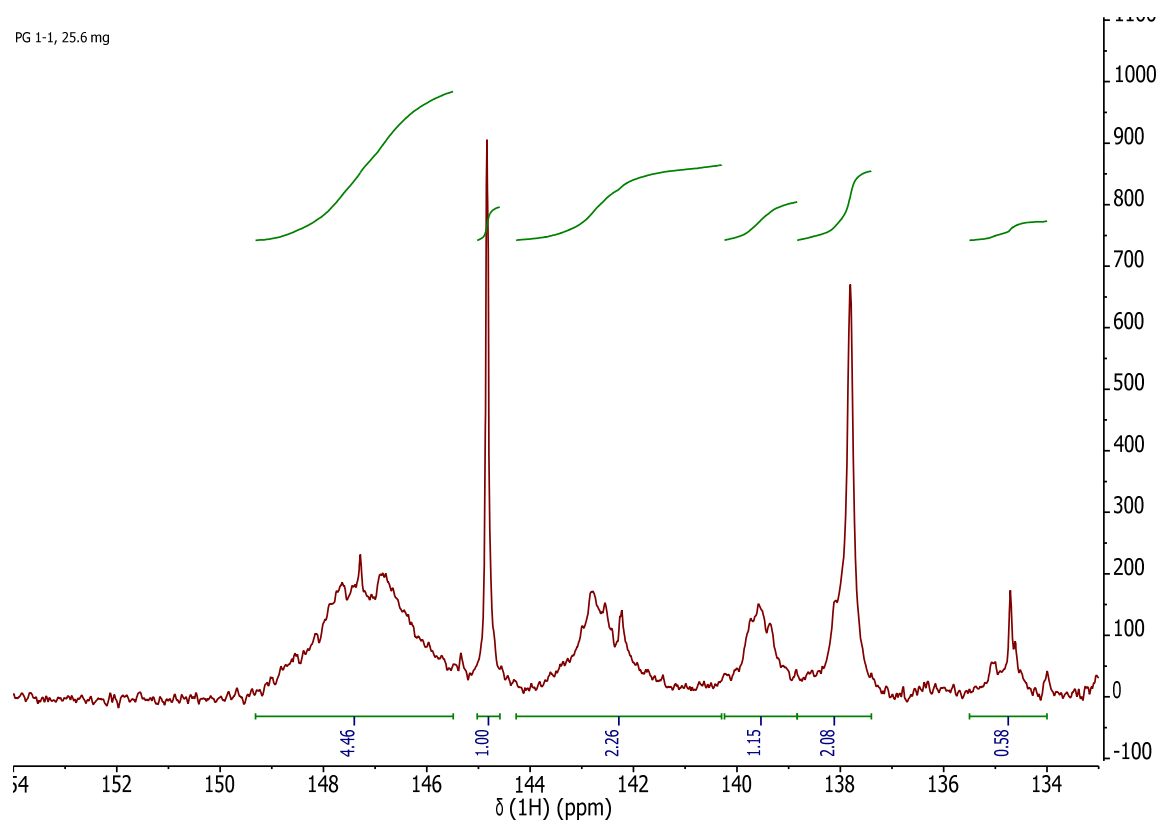

\section{CS-OSL blank}

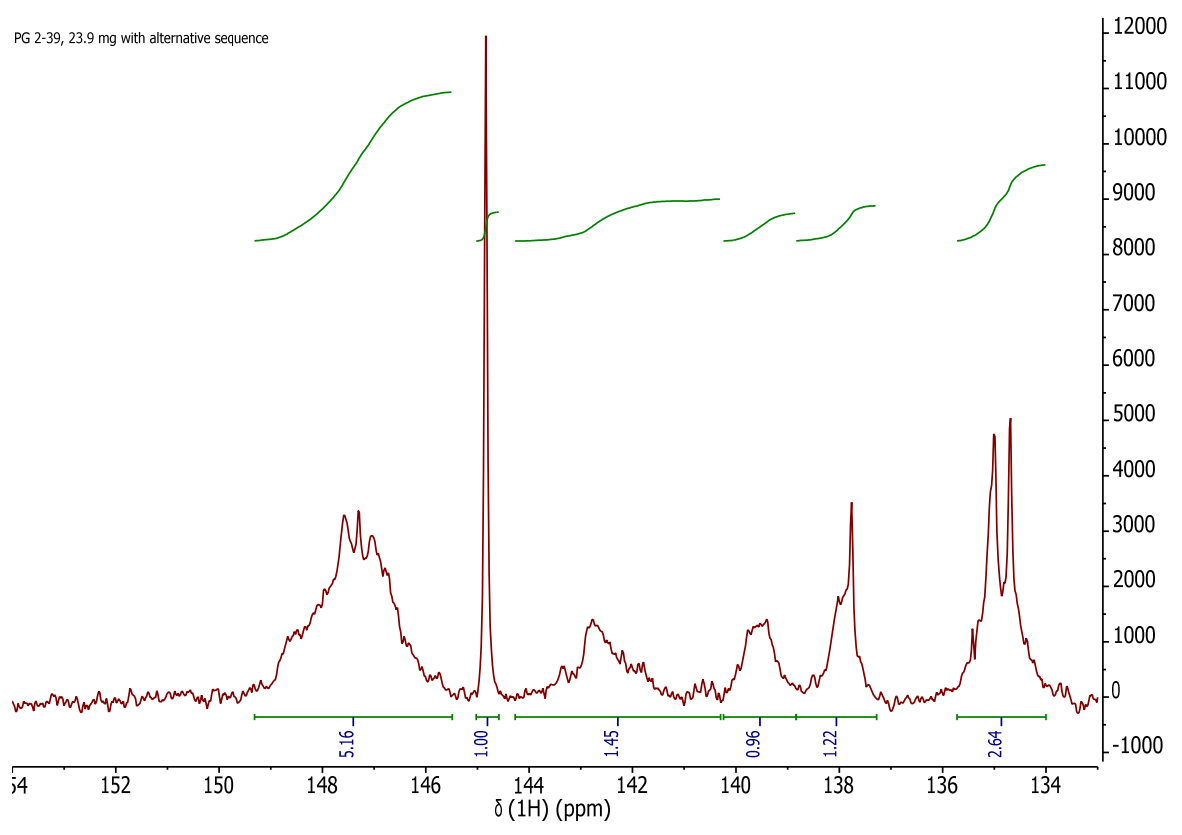


Table S2: Representative ${ }^{31}$ P NMR spectra for realised functionalised CS-OSL and CS-OSLbased copolymer functionalised either chemically or via enzymatic catalysis as described in the manuscript.

$\mathrm{CS}-\mathrm{OSL}+\mathrm{C}_{3}-\mathrm{NMe}_{3} \mathrm{Cl}(1.2 \mathrm{eq}).[$ Table 1, entry 14]

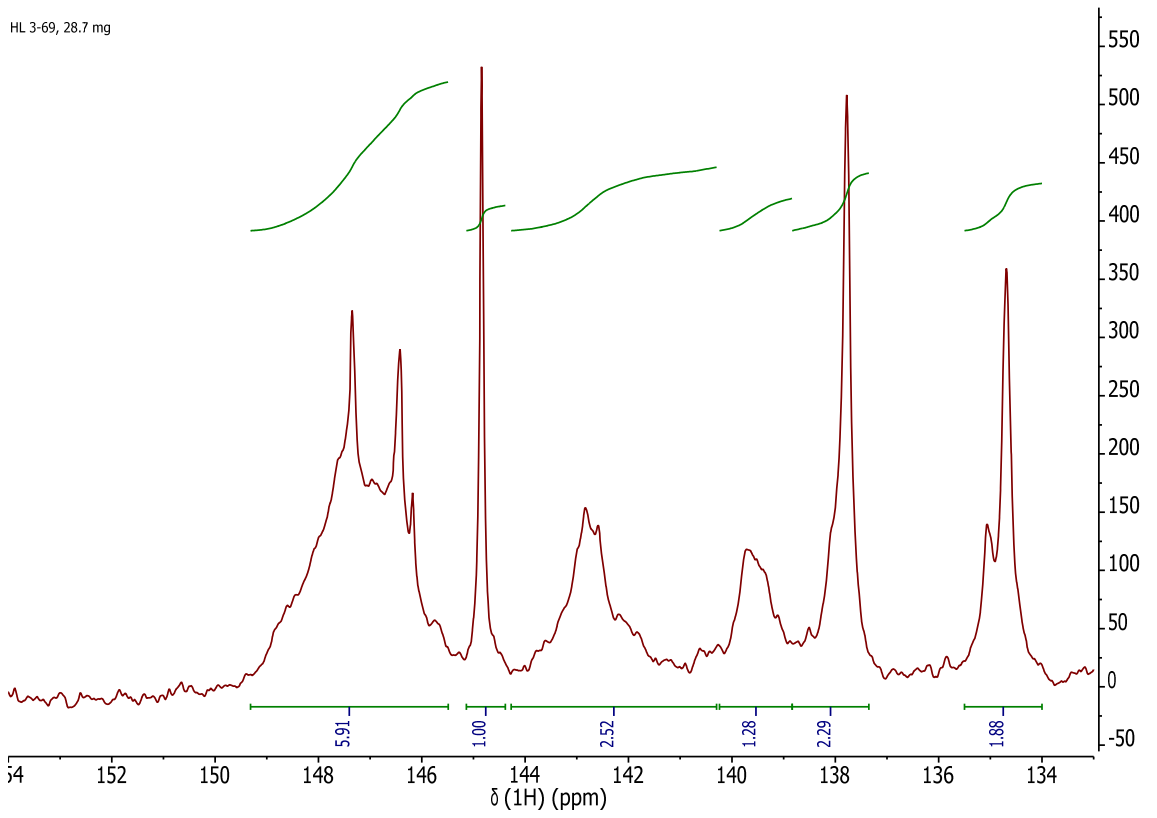

$\mathrm{CS}-\mathrm{OSL}+\mathrm{C}_{3}-\mathrm{CO}_{2} \mathrm{H}$ (1.2 eq.) [Table 1, entry 15]

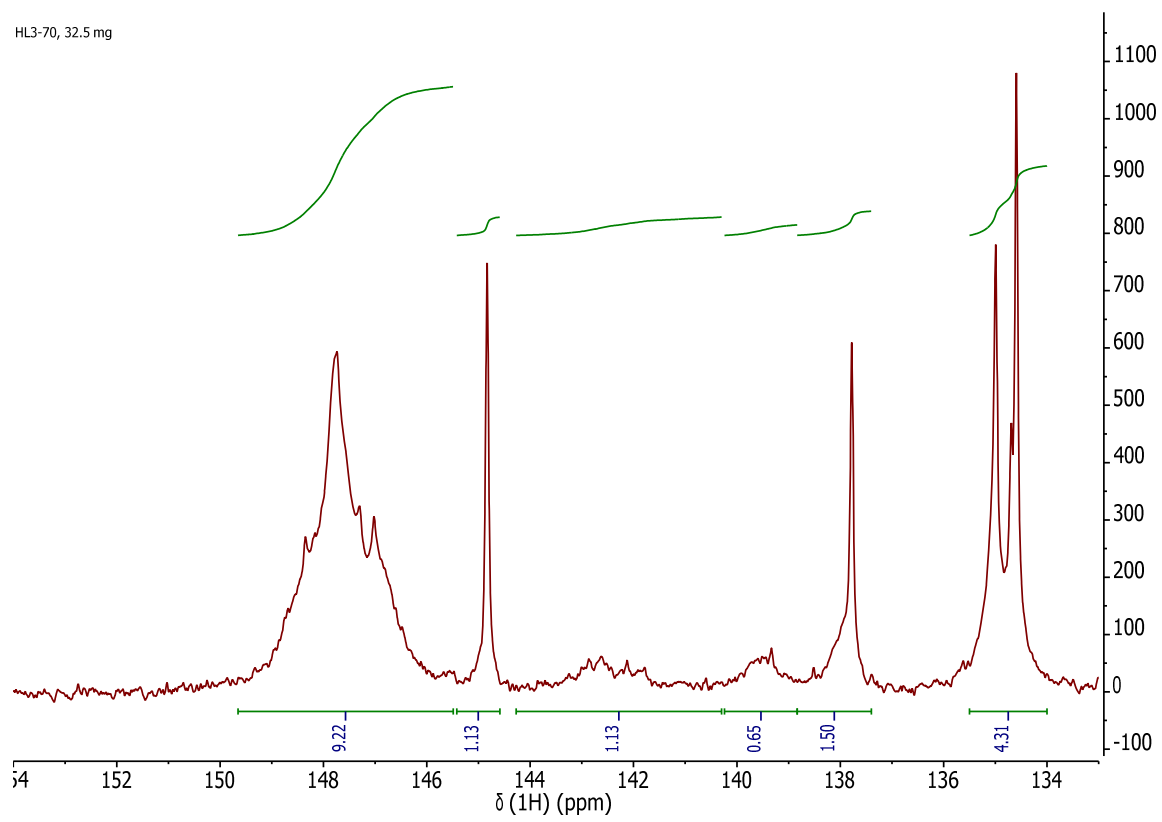


Table S2: Representative ${ }^{31}$ P NMR spectra for realised functionalised CS-OSL and CS-OSLbased copolymer functionalised either chemically or via enzymatic catalysis as described in the manuscript.

\section{CS-OSL + PDMS 5000 (1.0 eq.) [Table 2, entry 12]}

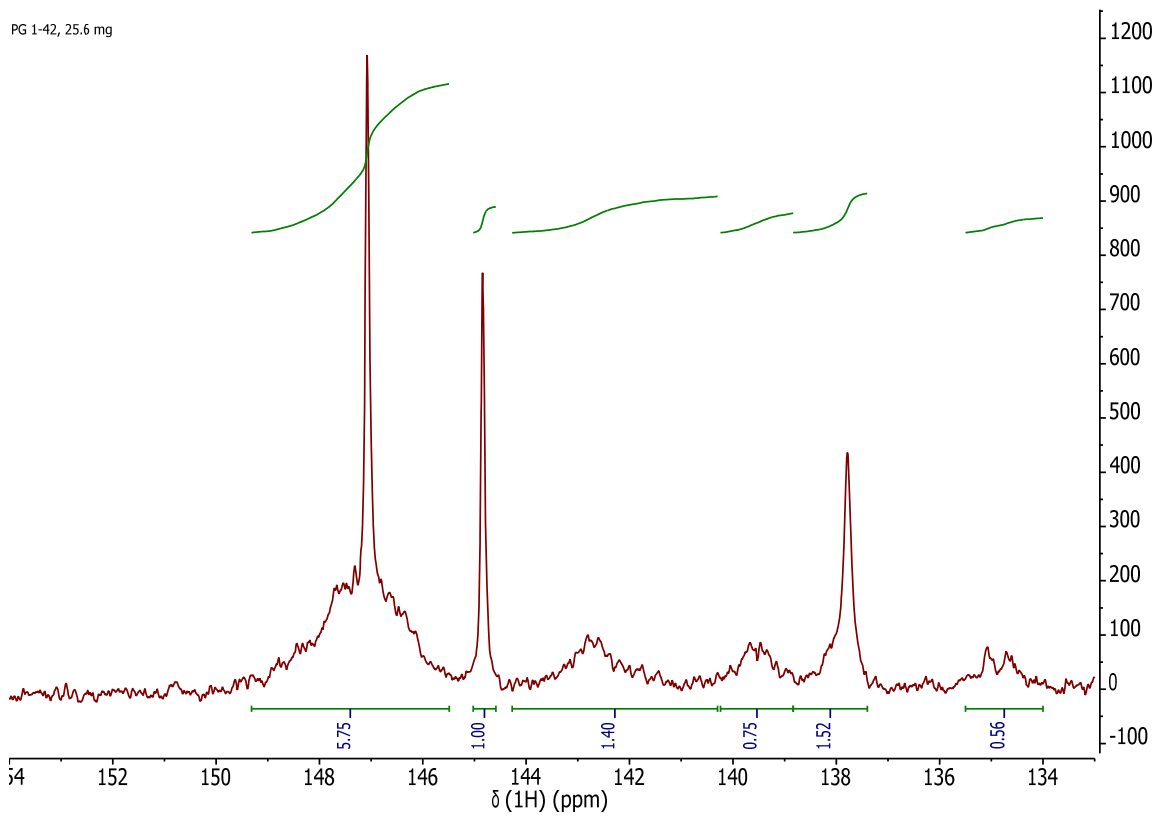

CS-OSL + PDMS 800 (1.1 eq.) [Table 2, entry 13]

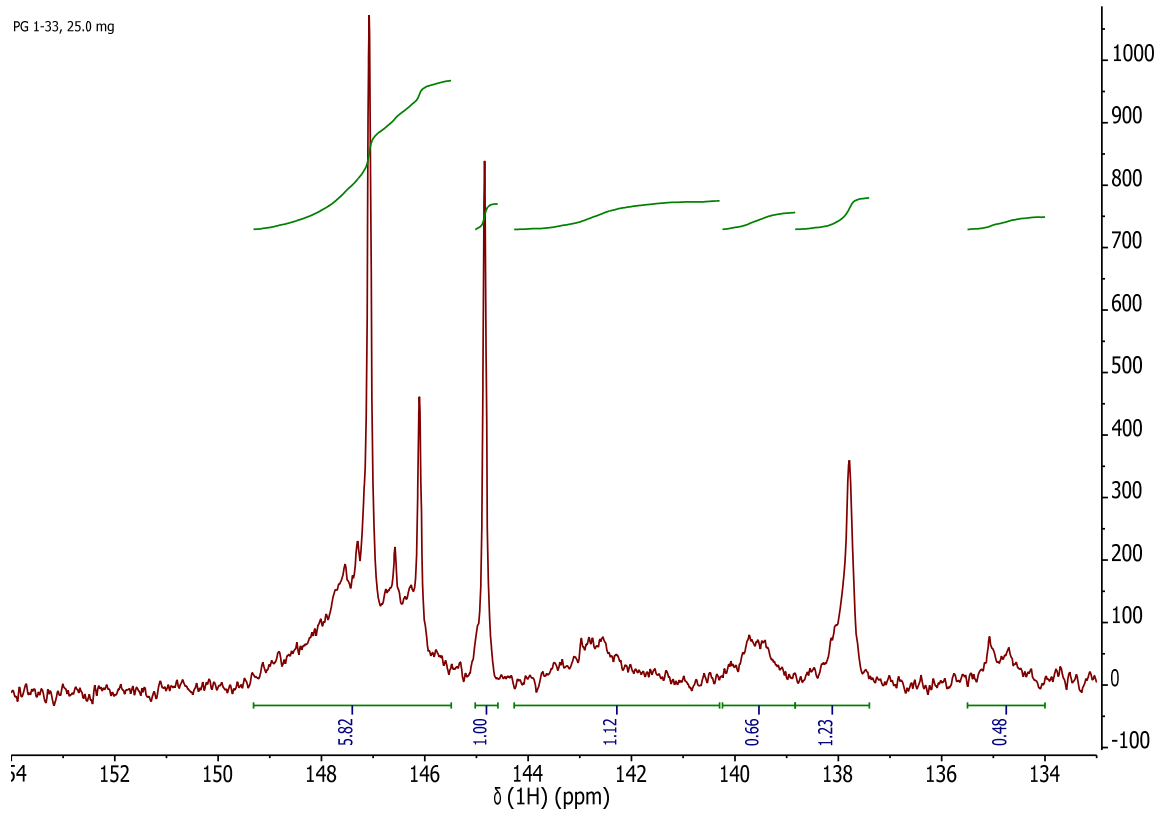


Table S2: Representative ${ }^{31}$ P NMR spectra for realised functionalised CS-OSL and CS-OSLbased copolymer functionalised either chemically or via enzymatic catalysis as described in the manuscript.

\section{CS-OSL + PDMS 5000 (1.0 eq.) [Table 3, entry 9]}

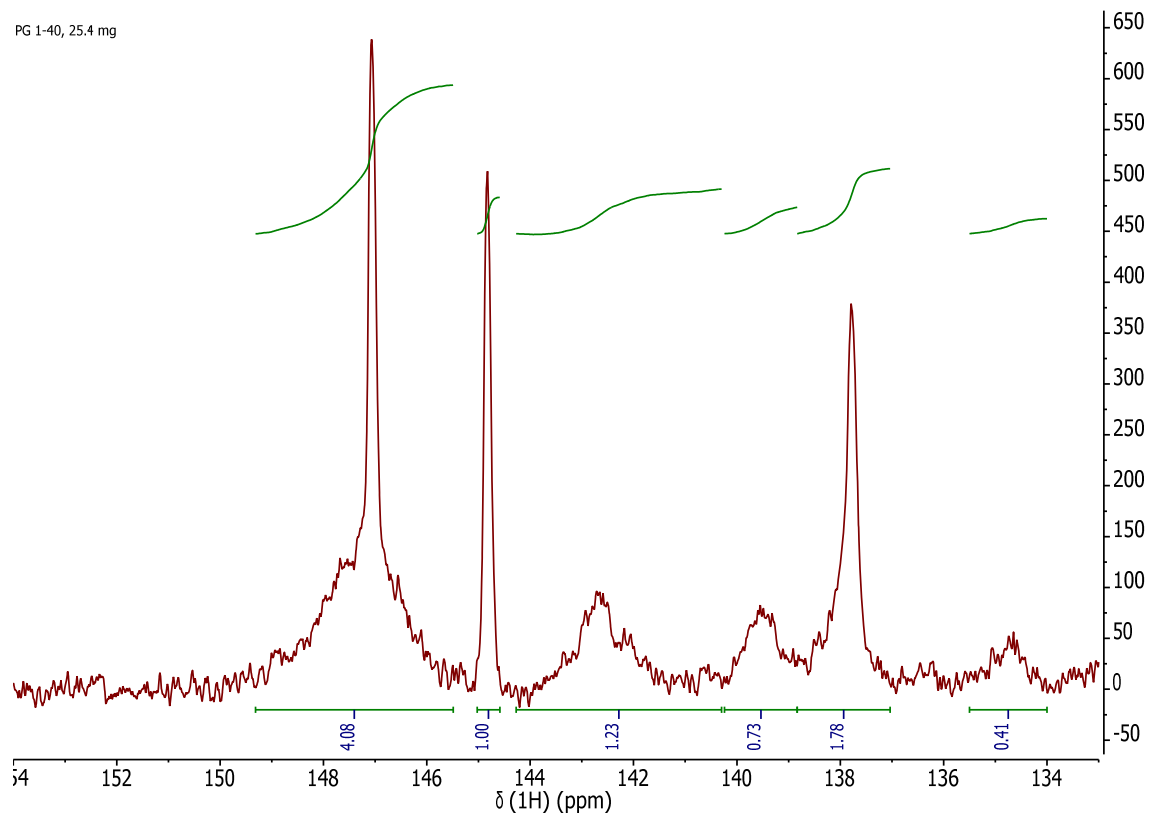

CS-OSL + PDMS ${ }_{800}(1.0$ eq.) [Table 3, entry 10]

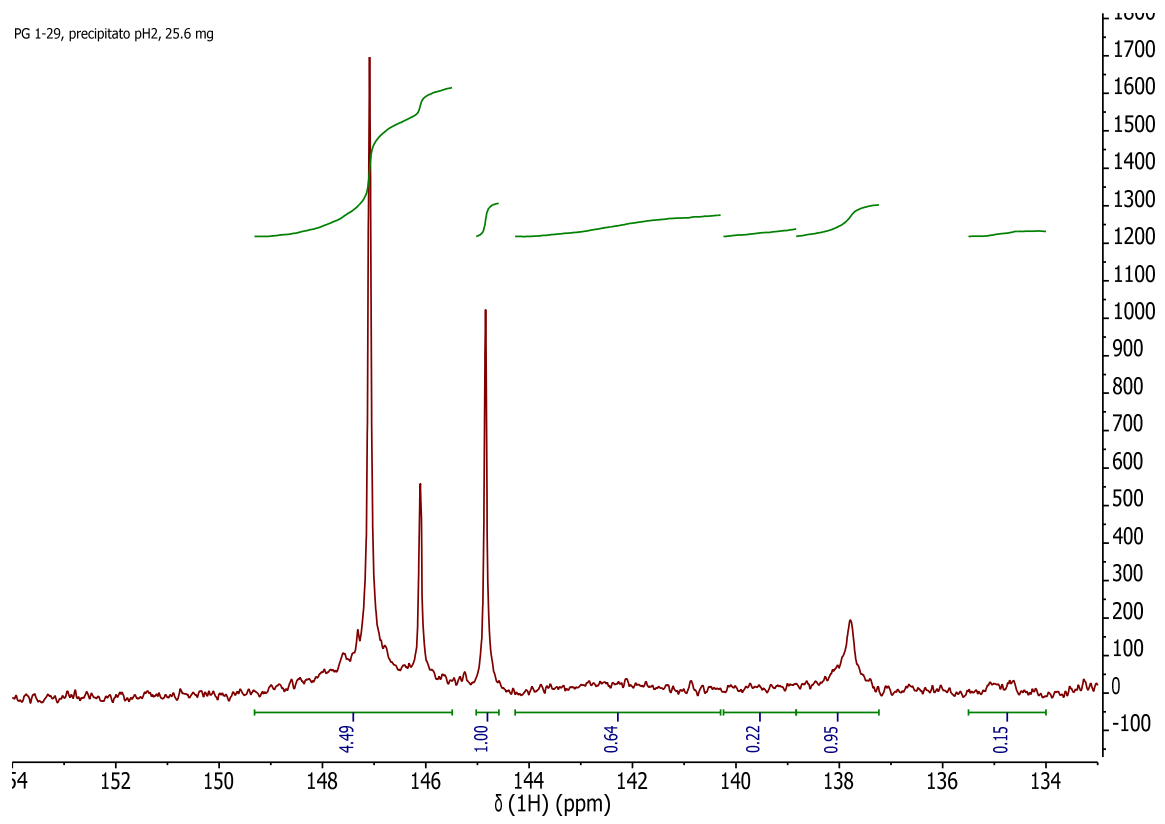


Table S2: Representative ${ }^{31}$ P NMR spectra for realised functionalised CS-OSL and CS-OSLbased copolymer functionalised either chemically or via enzymatic catalysis as described in the manuscript.

\section{CS-OSL + PEG 500 (0.1 eq.) [Table 3, entry 11]}

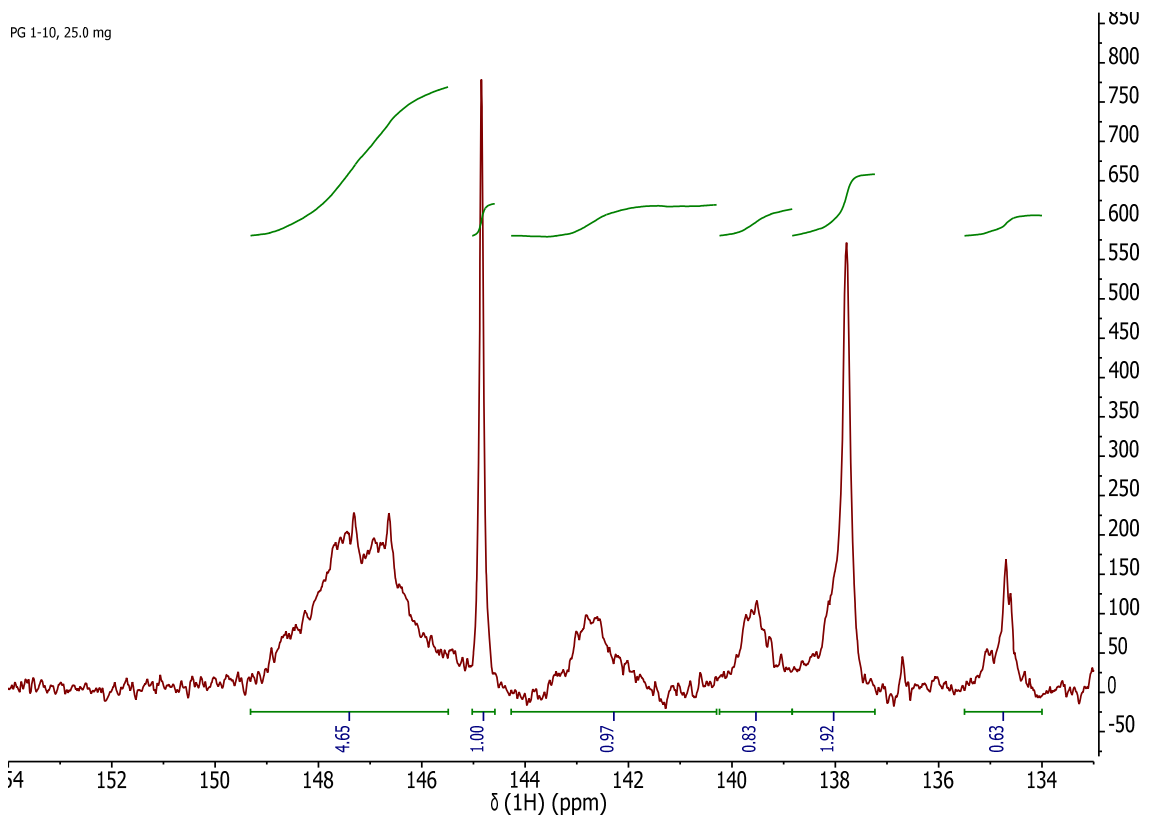

CS-OSL + PEG 500 (10 eq.) [Table 3, entry 12]

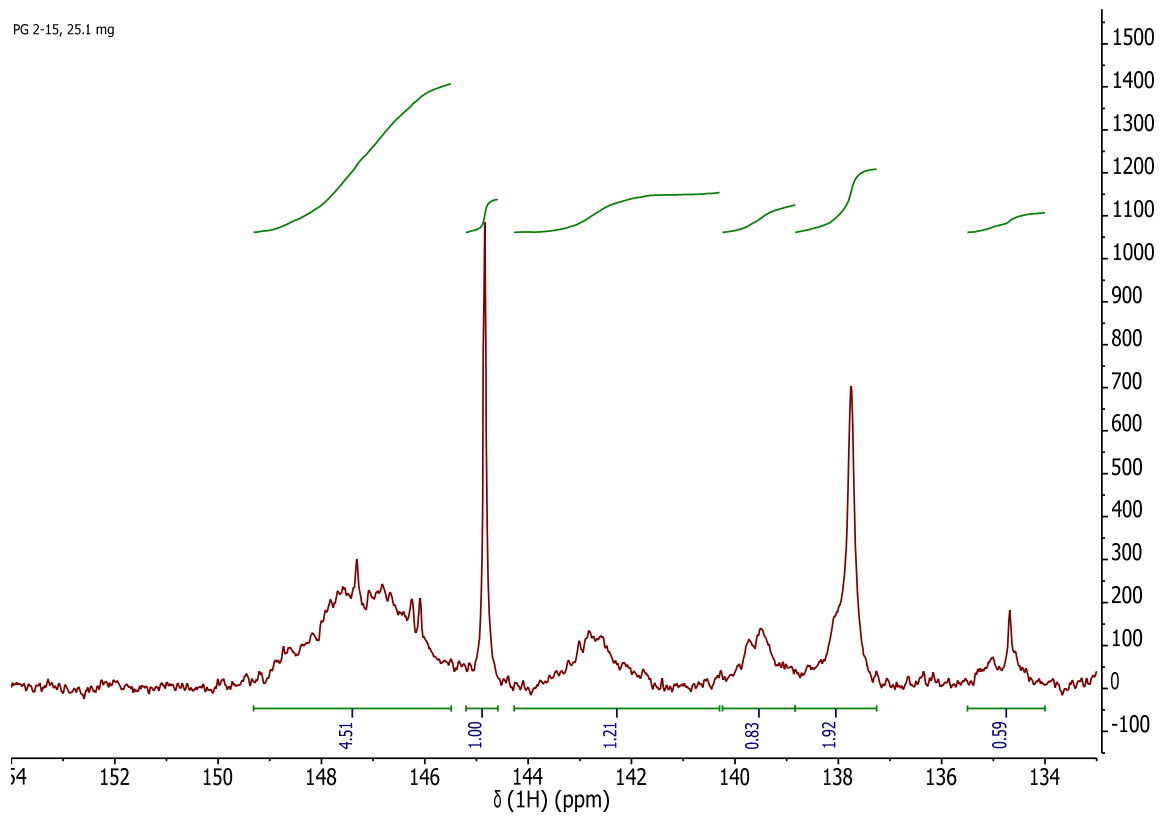

